\title{
Nonalcoholic fatty liver disease, a potential risk factor of non- specific ST-T segment changes: data from a cross-sectional study
}

\author{
Li Xiao ${ }^{\text {Equal first author, } 1}$, Tao Bai ${ }^{\text {Equal first author, } 1}{ }^{\text {, Junchao Zeng }}{ }^{2}$, Rui Yang ${ }^{\text {Corresp., }}{ }^{2}$, Ling Yang ${ }^{\text {Corresp. } 1}$ \\ 1 Division of Gastroenterology, Union Hospital, Tongji Medical College, Huazhong University of Science and Technology, Wuhan, China \\ 2 Physical Examination (Health Management $\square$ Center, Union Hospital, Tongji Medical College, Huazhong University of Science and Technology, Wuhan, \\ China \\ Corresponding Authors: Rui Yang, Ling Yang \\ Email address: 408025245@qq.com, hepayang@163.com
}

Background: Non-specific ST-T segment changes are prevalent and are proven risk factors for early onset of cardiovascular diseases. They can increase all-cause mortality by $100 \sim 200 \%$ and are candidate for early signs of cardiovascular changes. Nonalcoholic fatty liver disease (NAFLD) is prevalent worldwide and is one facet of a multisystem disease that confers substantial increases morbidity and mortality of nonalcoholic fatty liver-related cardiovascular diseases. It is unclear whether NAFLD is associated with non-specific ST-T changes warning early signs of cardiovascular changes. Therefore, we investigated this association. Methods: A cross-sectional study was designed that included a sample consisting of 32,922 participants who underwent health examinations. Participants with missing information, excessive alcohol intake, viral hepatitis, chronic liver disease or established cardiovascular diseases were excluded. Electrocardiograms were used for analysis of non-specific ST-T segment changes. NAFLD was diagnosed by ultrasonographic detection of hepatic steatosis without other liver diseases. A multivariable logistic regression model was served to calculate the OR and $95 \% \mathrm{Cl}$ for non-specific ST-T segment changes. Results: The prevalence of non-specific ST-T segment changes was $6.5 \%$ in participants with NAFLD, however, the prevalence of NAFLD was $42.9 \%$ in participants with non-specific ST-T segment changes. NAFLD was independently associated with nonspecific ST-T segment changes (OR: 1.925, 95\% Cl: 1.727-2.143, $P<0.001$ ). After adjusting for age, sex, heart rate, hypertension, body mass index, fasting glucose, total cholesterol, triglycerides, HDL-C, NAFLD remained an independent risk factor of nonspecific ST-T segment changes (OR: 1.289, 95\% Cl: 1.122-1.480). Conclusion: Nonspecific ST-T segment changes were independently associated with the presence of NAFLD after adjusting for potential confounders. 
1 Nonalcoholic fatty liver disease, a potential risk factor of non-specific ST-T segment changes: data from a cross-sectional study

\section{Authors' email addresses:}

LiXiao:LiXiao5366@163.com

Tao Bai: drbaitao@126.com

Junchao Zeng: 910065801@qq.com

Rui Yang: 408025245@qq.com

Ling Yang: hepayang@163.com; yanglinguh@hust.edu.cn

* Corresponding authors: Rui Yang, Physical Examination (Health Management)Centre, Union Hospital, Tongji Medical College, Huazhong University of Science and Technology, 1277 Jiefang Avenue, Wuhan, 430022, China, Email: 408025245@qq.com, phone +86-027-85726427; Ling Yang, M.D., Ph.D., Division of Gastroenterology, Union Hospital, Tongji Medical College, Huazhong University of Science and Technology, 1277 Jiefang Avenue, Wuhan, 430022, China, Email: hepayang@163.com, lingyang70@gmail.com, phone +86-027-85726678

\section{Abstract}

Background: Non-specific ST-T segment changes are prevalent and are proven risk factors for early onset of cardiovascular diseases. They can increase all-cause mortality by $100 \sim 200 \%$ and 
are candidate for early signs of cardiovascular changes. Nonalcoholic fatty liver disease (NAFLD) is prevalent worldwide and is one facet of a multisystem disease that confers substantial increases morbidity and mortality of nonalcoholic fatty liver-related cardiovascular diseases. It is unclear whether NAFLD is associated with non-specific ST-T changes warning early signs of cardiovascular changes. Therefore, we investigated this association.

Methods: A cross-sectional study was designed that included a sample consisting of 32,922 participants who underwent health examinations. Participants with missing information, excessive alcohol intake, viral hepatitis, chronic liver disease or established cardiovascular diseases were excluded. Electrocardiograms were used for analysis of non-specific ST-T segment changes. NAFLD was diagnosed by ultrasonographic detection of hepatic steatosis without other liver diseases. A multivariable logistic regression model was served to calculate the OR and 95\% CI for non-specific ST-T segment changes.

Results: The prevalence of non-specific ST-T segment changes was $6.5 \%$ in participants with NAFLD, however, the prevalence of NAFLD was $42.9 \%$ in participants with non-specific ST-T segment changes. NAFLD was independently associated with non-specific ST-T segment changes (OR: 1.925, 95\% CI: 1.727-2.143, $P<0.001$ ). After adjusting for age, sex, heart rate, hypertension, body mass index, fasting glucose, total cholesterol, triglycerides, HDL-C, NAFLD remained an independent risk factor of non-specific ST-T segment changes (OR: 1.289, 95\% CI: 1.122-1.480).

Conclusion: Non-specific ST-T segment changes were independently associated with the presence of NAFLD after adjusting for potential confounders.

\section{Introduction}

Nonalcoholic fatty liver disease (NAFLD) has emerged as the most frequent chronic liver disease worldwide (Patel SS \& Siddiqui MS, 2019), ranging from simple steatosis to steatohepatitis, cirrhosis, and hepatocellular cancer (Fuchs CD et al., 2014; Targher Giovanni, Day Christopher P. \& Bonora Enzo, 2010). Meanwhile, cardiovascular disease (CVD) is the most common cause of death, and its prevalence is constantly increasing worldwide (Balakumar P, Maung UK \& 
Jagadeesh G, 2016). Many studies have shown that NAFLD increases not only the risk of morbidity and mortality of liver-related cardiovascular disease, but also independently increases the risk for multi-vessel CVD associated with cardiovascular events (Anstee QM, Targher G \& Day CP, 2013; Boddi M et al., 2013; Chang Y et al., 2019; Targher G., Day C. P. \& Bonora E., 2010). Furthermore, a large body of literature currently demonstrates the association between NAFLD and cardiac arrhythmias; in particular, NAFLD is associated with an increased risk of atrial fibrillation, QT interval prolongation and ventricular tachyarrhythmia (Hung CS et al., 2015; Mantovani A. et al., 2016; Targher G et al., 2013; Targher G. et al., 2014). Therefore, it is urgent to early detect nonalcoholic fatty liver-related cardiovascular diseases.

Non-specific ST-T segment (NST) changes are characterized by a slightly upswept ST-segment depression or downward sloping and flat, diphasic or inverted $\mathrm{T}$ waves on a resting 12-lead electrocardiogram (ECG) (Kumar A et al., 2008). In clinical practice, such abnormalities are frequently encountered but generally ignored or simply considered to be incidental, transient, and benign electrocardiographic changes (Greenland P et al., 2003; Kumar A \& Lloyd-Jones DM, 2007). In addition, diverse morphological ECG abnormalities complicate the reasonable explanation of NST changes (Rautaharju PM et al., 2009). Physicians previously treated NST alterations as an unremarkable change. However, studies have shown that the all-cause mortality is increased by two to three times in patients with NST changes (Badheka AO et al., 2012). Furthermore, minor abnormalities have been reported to be independent risk factors for early CVD events and incident coronary heart disease (CHD) in middle-aged and older individuals, and the degree of risk increase is similar to that of elevated levels of traditional CVD risk factors, such as diabetes mellitus, hypercholesterolemia, and hypertension (Greenland P et al., 2003; Healy CF \& Lloyd-Jones DM, 2016; Kumar A et al., 2008; Rivero D et al., 2019; Walsh JA, 3rd et al., 2013). The existing evidence suggests that NST changes may be overlooked early signs of cardiovascular changes.

NAFLD is one facet of a multisystem metabolic abnormality. Previous studies have indicated that NAFLD could indicate an early stage of change during the process of CVD. Because it is 
81 important to predict and identify of the onset of CVD earlier, we aimed to evaluate the association between NAFLD and NST changes, hoping for greater vigilance for early prevention of CVD events, especially, in patients with NAFLD.

\section{Methods}

\section{Participants}

A total of 64,922 recordings were retrospectively reviewed at the physical examination centre in Wuhan Union Hospital from January 2017 to December 2018. All the participants were company staff or workers who came for annual health examination. $68.5 \%$ participants from the local company in Wuhan, 18\% from the other city outside of Wuhan in Hubei province and 14.5\% from other provinces. For the current cross-sectional study, we excluded 32,000 subjects for the following reasons: (1) aged $<18$ years or $>65$ years $(n=3667),(2)$ missing information on ECG, ultrasonography, or important covariates including body mass index (BMI), blood pressure (BP), fasting plasma glucose (FPG), liver function, and blood lipids $(n=25,036)$, (3) patients with excessive alcohol intake, viral hepatitis, chronic liver disease, or CVD $(n=3297)$. Some participants met more than one exclusion criterion, leaving 32,922 participants included in the final analysis (Figure 1). Tongji Medical College, Huazhong University of Science and Technology granted Ethical approval to carry out the study within its facilities (2018 S426). This study was for retrospective analysis only, so informed consent was waived.

\section{Clinical and laboratory data}

The BMI was calculated as weight divided by height squared $\left(\mathrm{kg} / \mathrm{m}^{2}\right)$. Blood pressure was recorded with a mercury sphygmomanometer in a sitting position. Participants were considered to be hypertensive if their systolic blood pressure was $\geq 140 \mathrm{~mm} \mathrm{Hg}$ or/and their diastolic blood pressure was $\geq 90 \mathrm{~mm} \mathrm{Hg}$. Fasting blood samples were drawn from the antecubital vein. Blood tests included fasting plasma glucose (FPG), total cholesterol (TC), triglycerides (TG), high-density lipoprotein (HDL-C), and low-density lipoprotein (LDL-C), alamine aminotransferase (ALT), 
108

109

110

111

112

113

114

115

116

117

118

119

120

121

122

123

124

125

126

127

128

129

130

131

132

133

134

aspartate aminotransferase (AST), alkaline phosphatase (ALP), gamma-glutamyl transferase (GGT).

\section{Hepatic ultrasonography}

Hepatic ultrasonic examinations were performed in all participants by experienced ultrasonographers who were blinded to the clinical and laboratory data. Hepatic steatosis was determined based on standard criteria, including the presence of a diffuse increase in echogenicity compared to the cortex of the ipsilateral kidney, posterior attenuation of the ultrasound beam, and poor visualization of the walls of the portal vein (Chang Y et al., 2019). The severity of hepatic steatosis was also recorded as no (less than $5 \%)$, mild $(\geq 5-33 \%)$, or moderate $(>33-66 \%)$ and severe $(\geq 66 \%)$ on sonography. Because the number of severe steatosis was too small, the steatosis of NAFLD was classified as absent, mild and moderate/severe.

\section{ECG and non-specific ST-T segment changes}

A standard 12-lead electrocardiogram (ECG) was recorded for detection of NST changes, which were specifically defined according to Minnesota Codes 4-3, 4-4, 5-3, and 5-4 (Prineas RJ C, R. S. \& Zhang, Z.-M, 2010).

\section{Statistical analysis}

Data are expressed as the mean \pm standard deviation (SD), median (interquartile range) or proportion. An unpaired t-test, one-way ANOVA and the chi-squared test were used to compare differences among the clinical characteristics of the participants categorized by NST changes. Four regression models were performed to progressively reduce the impact of potential confounders on the association between NAFLD and NST changes. Basic model 1 was unadjusted; multivariable model 2 was adjusted for age and sex; model 3 included the heart rate, presence of hypertension (blood pressure $\geq 140 / 90 \mathrm{mmHg}$ ) and fasting plasma glucose plus the same variables included in model 2, and finally, the analysis was further adjusted for the same variables included in model 3 
135

136

137

138

139

140

141

142

143

144

145

146

147

148

149

150

151

152

153

154

155

156

157

158

159

160

161

plus BMI, TC, TG, and HDL-C (model 4). Covariates included in the multivariate regression models were chosen as potential confounders based on their biological plausibility or statistical association with NST changes in univariable analyses.

All statistical analyses were performed with SPSS 21.0, and all $P$ values were two-tailed. Values of $P<0.05$ were considered statistically significant.

\section{Results}

\section{Characteristics of participants}

The clinical and biochemical characteristics of participants stratified by NST changes were summarized in Table 1. NAFLD was present in $9454(28.7 \%)$ patients who underwent health examinations. The prevalence of non-specific ST-T segment changes was $6.5 \%$ in participants with NAFLD, however, the prevalence of NAFLD was $42.9 \%$ in participants with non-specific ST-T segment changes. The mean ages (SD) of patients with and without NST changes were 50 years and 41 years $(P<0.001)$, respectively, a higher prevalence presented in middle-aged and older individuals. The mean BMI (SD) was $23.4 \mathrm{~kg} / \mathrm{m}^{2}$ in patients without NST changes and 24.7 $\mathrm{kg} / \mathrm{m}^{2}$ in patients with NST changes, suggesting that overweight people may be more likely to have NST changes. Patients with NST changes tended to be female $(60.7 \%$ vs $39.3 \%$ in males, $P<$ 0.001). In patients with NST changes, the incidence of mild steatosis and moderate/severe steatosis were $35.3 \%$ and $7.6 \%$, respectively. In addition, NST changes were associated with blood pressure, and higher levels of fasting plasma glucose, triglycerides, total cholesterol, LDL-C, and lower levels of HDL-C.

\section{Association between NST changes and NAFLD}

Remarkably, as shown in Figure 2, the proportion of the patients with NST changes was associated with the presence of NAFLD on ultrasound $(P<0.001$ for the trend). In patients with no steatosis, mild steatosis and moderate/severe steatosis, the incidence of the NST changes were $3.49 \%, 6.59 \%$ and $6.17 \%$, respectively. However, the proportions of NST changes were not significantly different 
162 between patients with mild and moderate/severe steatosis.

163 As shown in Table 2, the univariate analysis indicated that the association between NAFLD and 164 NST changes was significant, with a 1.925-fold increased OR (95\% CI: 1.729-2.143) (model 1).

165 Our data suggested that NAFLD is an independent risk factor for NST changes. Adjustment for 166 age and sex did not detectably weaken the association between NAFLD and NST changes (model 167 2), the corresponding OR (95\% CI) remained 1.935 (95\% CI: 1.725-2.170). After further 168 adjustments for other potentially confounding factors including heart rate, hypertension (blood 169 pressure $\geq 140 / 90 \mathrm{mmHg}$ ), FPG, BMI, TC, TG, and HDL-C, NAFLD remained a significant risk 170 factor for NST changes, and the corresponding multivariable-adjusted OR was 1.289 (95\% CI: 171 1.122-1.480). In model 4, we found that age, BMI, hypertension, high FBG, and high TC were 172 also independent predictors of NST changes

173

174

175 176

\section{Association between NST changes and NAFLD in different subgroups}

To further elucidate the association between NAFLD and NST changes, we investigated the significance of differences in subgroups stratified by sex, age, BMI, hypertension, fasting glucose, HDL-C, and total cholesterol (Figure 3). The subgroup analyses indicated that the association between NAFLD and NST changes was stronger in males (OR 1.449, 95\% CI: 1.178 1.781) than in females (OR: 1.161, 95\% CI: 0.958 1.406). Likewise, differences were larger for the 3rd and 4th quartile of age than for the other two quartiles. However, similarly large impacts were observed across participant subgroups for normal BMI or overweight, no hypertension and hypertension. Interesting, it was less relevant in high fasting glucose, low HDL-C and high TC than their respectively references.

\section{Discussion}

In this large-sample retrospective study including records of health examinations, NST changes were significantly associated with NAFLD, notably, the association was independent of potential confounders. In addition, we found that age, sex, hypertension, fasting glucose, TC and HDL-C 
189 levels might be significant predictors of NST changes. Our findings suggested that the NST

changes might represent an additional correlation with higher CVD risk in patients with NAFLD, and emphasized the importance of evaluating arrhythmic risk in NAFLD patients so as to strengthen early prevention and early intervention of CVD.

Previous studies have demonstrated that there existed significant correlation between NAFLD and cardiac arrhythmias (Ismaiel A et al., 2019), such as QTc prolongation (Targher Giovanni et al., 2014), atrial fibrillation (Anstee QM et al., 2018), ventricular arrhythmias (Mantovani Alessandro et al., 2016), but little attention was paid to explore the relationship between NST and NAFLD. Although, it has been suggested that NAFLD have adverse impact on ST segment elevation in myocardial infarction (Emre Ayse et al., 2015; Keskin M et al., 2017) and NAFLD was correlated with silent myocardial ischemia (Lee S et al., 2006), NST changes may be earlier signs of cardiovascular changes (Healy CF \& Lloyd-Jones DM, 2016). The present study provided evidence indicating that NST changes were significantly associated with NAFLD, which heightened greater vigilance for early prevention of CVD events in patients with NAFLD. However, intriguingly, we found that the incidence of NST in the moderate/severe hepatic steatosis was slightly lower $(6.17 \%)$ compared to the incidence of NST in patients with mild steatosis $(6.59 \%)$. We believed the reason was that the population we studied was made up of relatively healthy people under 65 years old undergoing health checkup, and the number of severe steatosis was very few in these patients. We need to further investigate the relationship between NST and NAFLD in the elderly patients and the hospitalized patients.

Consistent with our results, previous studies have reported the risk factors of NST changes, such as age, sex, hypertension, and serum triglyceride levels. The prevalence of NST abnormalities was higher in females $(4.5 \%$ vs $2.3 \%$ in males; $P<0.001)$ among healthy volunteers (Hingorani $\mathrm{P}$ et al., 2012), and similar findings were also observed in an Australian Busselton study (Cullen K et al., 1982) and the Michigan study (Ostrander LD, Jr. et al., 1965). One study proposed that NST changes were associated with a remarkably higher incidence rate of unsatisfactory blood pressure control in hypertension patients, especially among diabetic patients (Bao H et al., 2017). Kumar et 
216

217

218

219

220

221

222

223

224

225

226

227

228

229

230

231

232

233

234

235

236

237

238

239

240

241

242

al. also concluded that patients with high blood pressure tended to have NST changes (Kumar A et al., 2008). Similarly, an epidemiologic cohort study suggested that the incidence rate of resting ST-T segment abnormalities in men without CHD is greatly affected by age, increasing from $2 \%$ at 40 years to $30 \%$ at the age of 80 years. Men with such ST-T segment changes were older and had higher serum triglyceride levels and worse glucose tolerance (Sigurdsson E et al., 1996). In addition, in line with LDL-C as an independent risk factor for CVD (Moriarty PM, Gray JV \& Gorby LK, 2019), our result also substantiated that LDL-C was implicated in NST changes. These previous studies validated our results and further provided insights regarding the potential predictive value of NST changes associated with NAFLD. NST changes associated with NAFLD might reveal an additional connection to increased CVD risk in patients with NAFLD and emphasize the importance of evaluating the cardiovascular and arrhythmic risk for early warning and prevention in those patients.

Although NST changes are significantly associated with NAFLD, the pathophysiological mechanisms have not been fully elucidated. Here, we postulated that the possible mechanisms include the following. First, NST changes might indicate subclinical CVD, early left ventricular hypertrophy, or increased left ventricular mass (Kumar A \& Lloyd-Jones DM, 2007). NAFLD might be related to NST due to shared risk factors for cardiac metabolism and co-morbidities or as a marker of coexisting ectopic accumulation of fat in other organs. For example, myocardial steatosis and accumulated pericardial fat might result in myocardial functional and structural disturbances (Fox CS et al., 2009; Hallsworth K et al., 2013). Second, myocardial perfusion is more vulnerable to impairment when the NAFLD score is $\geq 3$ (Emre A. et al., 2015); coincidentally, NST abnormalities were associated with impaired coronary circulation in a healthy population (Larsen CT et al., 2002). Accumulating evidence suggests that hepatic steatosis may aggravate systemic insulin resistance and alter secretory patterns of hepatokines and proatherogenic factors that may play a role in the pathophysiology of NST changes (Bhatia LS et al., 2012). Such an association among NST changes, cardiac function and coronary circulation offers a plausible interpretation for the association between NST changes and NAFLD in our study. 
243 Despite these assumptions, there is no conclusive data to link NST changes with any specific 244 physiological mechanism in patients with NAFLD. Further study is needed to clarify the 245 pathophysiologic association between NST changes and NAFLD.

246 There are some limitations of this study. First, this is a cross-sectional study in which the 247 participants were screened based on the inclusion and exclusion criteria, thus leading to difficultly 248 in determining causal relationships between NAFLD and NST changes (Setia MS, 2016). Second, 249 NAFLD was diagnosed based on US, which is less sensitive when liver fat infiltration is less than 250 approximately $30 \%$. However, this method is potentially a practical tool in clinical practice 251 because of its relatively low cost, non-invasive nature and diagnostic accuracy. Finally, residual 252 confounders were not eliminated due to the measurement errors of these variables. Despite these 253 limitations, our study selected a large sample of healthy individuals who were less likely to be affected by comorbidity-related biases than those in studies conducted in higher risk populations.

\section{Conclusion}

257 Our study suggested that NST changes were significantly and independently associated with NAFLD after adjusting for potential confounding factors. Further studies are required to investigate the relationship between NST changes and NAFLD in the elderly patients and the hospitalized patients and elucidate the pathophysiological mechanisms and determine the longterm clinical effects of NAFLD on NST changes.

\section{List of Abbreviations}

Nonalcoholic fatty liver disease: NAFLD; NST: non-specific ST-T segment; ECG: Electrocardiogram; OR: odds ratio; SD: standard deviation; CI: confidence interval; CVD: cardiovascular disease; CHD: coronary heart disease; BP: blood pressures; FPG: fasting plasma glucose; BMI: body mass index; HDL-C: high density lipoprotein cholesterol; TC: total cholesterol; TG: triglyceride; LDL-C: low density lipoprotein cholesterol; ALT: alamine aminotransferase, AST: aspartate aminotransferase, ALP: alkaline phosphatase, GGT: gammaglutamyl transferase, US: ultrasonography 
Acknowledgments:

273 We thank all of the enrolled participants for their contribution to this study.

274

275

276

277

278

279

280

281

282

283

\section{References}

Anstee QM, Mantovani A, Tilg H, Targher GJNRG, \& Hepatology. 2018. Risk of cardiomyopathy and cardiac arrhythmias in patients with nonalcoholic fatty liver disease. 15 (7): 425-439.

Anstee QM, Targher G, \& Day CP. 2013. Progression of NAFLD to diabetes mellitus, cardiovascular disease or cirrhosis. Nature Reviews: Gastroenterology \& Hepatology. 10 (6): 330-344. DOI:10.1038/nrgastro.2013.41.

Badheka AO, Rathod A, Marzouka GR, Patel N, Bokhari SS, Moscucci M, \& Cohen MG. 2012. Isolated nonspecific ST-segment and T-wave abnormalities in a cross-sectional United States population and Mortality (from NHANES III). American Journal of Cardiology. 110 (4): 521 525. DOI:10.1016/j.amjcard.2012.04.023.

Balakumar P, Maung UK, \& Jagadeesh G. 2016. Prevalence and prevention of cardiovascular disease and diabetes mellitus. Pharmacological Research. 113 (Pt A): 600-609. DOI:10.1016/j.phrs.2016.09.040.

Bao H, Cai H, Zhao Y, Huang X, Fan F, Zhang C, Li J, Chen J, Hong K, Li P, Wu Y, Wu Q, Wang B, Xu X, Li Y, Huo Y, \& Cheng X. 2017. Nonspecific ST-T changes associated with unsatisfactory blood pressure control among adults with hypertension in China: Evidence from the CSPTT study. Medicine (Baltimore). $96 \quad$ (13): $\quad$ e6423. DOI:10.1097/MD.0000000000006423.

Bhatia LS, Curzen NP, Calder PC, \& Byrne CD. 2012. Non-alcoholic fatty liver disease: a new and important cardiovascular risk factor? European Heart Journal. 33 (10): 1190-1200. DOI:10.1093/eurheartj/ehr453.

Boddi M, Tarquini R, Chiostri M, Marra F, Valente S, Giglioli C, Gensini GF, \& Abbate R. 2013. Nonalcoholic fatty liver in nondiabetic patients with acute coronary syndromes. European Journal of Clinical Investigation. 43 (5): 429-438. DOI:10.1111/eci.12065. 
299

300

301

302

303

304

305

306

307

308

309

310

311

312

313

314

315

316

317

318

319

320

321

322

323

324

325

Chang Y, Ryu S, Sung KC, Cho YK, Sung E, Kim HN, Jung HS, Yun KE, Ahn J, Shin H, Wild SH, \& Byrne CD. 2019. Alcoholic and non-alcoholic fatty liver disease and associations with coronary artery calcification: evidence from the Kangbuk Samsung Health Study. Gut. 68 (9): 1667-1675. DOI:10.1136/gutjnl-2018-317666.

Cullen K, Stenhouse NS, Wearne KL, \& Cumpston GN. 1982. Electrocardiograms and 13 year cardiovascular mortality in Busselton study. British Heart Journal. 47 (3): 209-212. DOI:10.1136/hrt.47.3.209.

Emre A, Terzi S, Celiker E, Sahin S, Yazici S, Erdem A, Ceylan US, Asik M, \& Yesilcimen K. 2015. Impact of Nonalcoholic Fatty Liver Disease on Myocardial Perfusion in Nondiabetic Patients Undergoing Primary Percutaneous Coronary Intervention for ST-Segment Elevation Myocardial Infarction. American Journal of Cardiology. 116 (12): 1810-1814. DOI:10.1016/j.amjcard.2015.09.021.

Emre A, Terzi S, Celiker E, Sahin S, Yazıcı S, Erdem A, Ceylan US, Asik M, \& Yesilcimen KJTAjoc. 2015. Impact of nonalcoholic fatty liver disease on myocardial perfusion in nondiabetic patients undergoing primary percutaneous coronary intervention for ST-segment elevation myocardial infarction. 116 (12): 1810-1814.

Fox CS, Gona P, Hoffmann U, Porter SA, Salton CJ, Massaro JM, Levy D, Larson MG, D'Agostino RB, Sr., O'Donnell CJ, \& Manning WJ. 2009. Pericardial fat, intrathoracic fat, and measures of left ventricular structure and function: the Framingham Heart Study. Circulation. 119 (12): 1586-1591. DOI:10.1161/circulationaha.108.828970.

Fuchs CD, Claudel T, Trauner MJTiE, \& Metabolism. 2014. Role of metabolic lipases and lipolytic metabolites in the pathogenesis of NAFLD. 25 (11): 576-585.

Greenland P, Xie X, Liu K, Colangelo L, Liao Y, Daviglus ML, Agulnek AN, \& Stamler J. 2003. Impact of minor electrocardiographic ST-segment and/or T-wave abnormalities on cardiovascular mortality during long-term follow-up. American Journal of Cardiology. 91 (9): 1068-1074.

Hallsworth K, Hollingsworth KG, Thoma C, Jakovljevic D, MacGowan GA, Anstee QM, Taylor 
326

327

328

329

330

331

332

333

334

335

336

337

338

339

340

341

342

343

344

345

346

347

348

349

350

351

352

R, Day CP, \& Trenell MI. 2013. Cardiac structure and function are altered in adults with nonalcoholic fatty liver disease. Journal of Hepatology. 58 (4): 757-762. DOI:10.1016/j.jhep.2012.11.015.

Healy CF, \& Lloyd-Jones DM. 2016. Association of Traditional Cardiovascular Risk Factors With Development of Major and Minor Electrocardiographic Abnormalities: A Systematic Review. Cardiology in Review. 24 (4): 163-169. DOI:10.1097/crd.0000000000000109.

Hingorani P, Natekar M, Deshmukh S, Karnad DR, Kothari S, Narula D, \& Lokhandwala Y. 2012. Morphological abnormalities in baseline ECGs in healthy normal volunteers participating in phase I studies. Indian Journal of Medical Research. 135: 322-330.

Hung CS, Tseng PH, Tu CH, Chen CC, Liao WC, Lee YC, Chiu HM, Lin HJ, Ho YL, Yang WS, Wu MS, \& Chen MF. 2015. Nonalcoholic Fatty Liver Disease Is Associated With QT Prolongation in the General Population. J Am Heart Assoc. 4 (7). DOI:10.1161/jaha.115.001820.

Ismaiel A, Colosi HA, Rusu F, \& Dumitrașcu DL. 2019. Cardiac Arrhythmias and Electrocardiogram Modifications in Non-Alcoholic Fatty Liver Disease. A Systematic Review. Journal of gastrointestinal and liver diseases : JGLD. 28 (4): 483-493. DOI:10.15403/jgld-344.

Keskin M, Hayıroğlu Mİ, Uzun AO, Güvenç TS, Şahin S, \& Kozan ÖJTAjoc. 2017. Effect of Nonalcoholic Fatty Liver Disease on In-Hospital and Long-Term Outcomes in Patients With ST-Segment Elevation Myocardial Infarction. 120 (10): 1720-1726.

Kumar A, \& Lloyd-Jones DM. 2007. Clinical significance of minor nonspecific ST-segment and T-wave abnormalities in asymptomatic subjects: a systematic review. Cardiology in Review. 15 (3): 133-142. DOI:10.1097/01.crd.0000249382.65955.14.

Kumar A, Prineas RJ, Arnold AM, Psaty BM, Furberg CD, Robbins J, \& Lloyd-Jones DM. 2008. Prevalence, prognosis, and implications of isolated minor nonspecific ST-segment and T-wave abnormalities in older adults: Cardiovascular Health Study. Circulation. 118 (25): 2790-2796. DOI:10.1161/circulationaha.108.772541.

Larsen CT, Dahlin J, Blackburn H, Scharling H, Appleyard M, Sigurd B, \& Schnohr P. 2002. 
353

354

355

356

357

358

359

360

361

362

363

364

365

366

367

368

369

370

371

372

373

374

375

376

377

378

379

Prevalence and prognosis of electrocardiographic left ventricular hypertrophy, ST segment depression and negative T-wave; the Copenhagen City Heart Study. European Heart Journal. 23 (4): 315-324. DOI:10.1053/euhj.2001.2774.

Lee S, Jin Kim Y, Yong Jeon T, Hoi Kim H, Woo Oh S, Park Y, \& Soo Kim SJSjog. 2006. Obesity is the only independent factor associated with ultrasound-diagnosed non-alcoholic fatty liver disease: a cross-sectional case-control study. 41 (5): 566-572.

Mantovani A, Rigamonti A, Bonapace S, Bolzan B, Pernigo M, Morani G, Franceschini L, Bergamini C, Bertolini L, Valbusa F, Rigolon R, Pichiri I, Zoppini G, Bonora E, Violi F, \& Targher G. 2016. Nonalcoholic Fatty Liver Disease Is Associated With Ventricular Arrhythmias in Patients With Type 2 Diabetes Referred for Clinically Indicated 24-Hour Holter Monitoring. Diabetes Care. 39 (8): 1416-1423. DOI:10.2337/dc16-0091.

Mantovani A, Rigamonti A, Bonapace S, Bolzan B, Pernigo M, Morani G, Franceschini L, Bergamini C, Bertolini L, \& Valbusa FJDC. 2016. Nonalcoholic fatty liver disease is associated with ventricular arrhythmias in patients with type 2 diabetes referred for clinically indicated 24-hour Holter monitoring. 39 (8): 1416-1423.

Moriarty PM, Gray JV, \& Gorby LK. 2019. Lipoprotein apheresis for lipoprotein(a) and cardiovascular disease. Journal of Clinical Lipidology. DOI:10.1016/j.jacl.2019.09.010.

Ostrander LD, Jr., Brandt RL, Kjelsberg MO, \& $\quad$ Epstein FH. 1965. ELECTROCARDIOGRAPHIC FINDINGS AMONG THE ADULT POPULATION OF A TOTAL NATURAL COMMUNITY, TECUMSEH, MICHIGAN. Circulation. 31: 888-898.

Patel SS, \& Siddiqui MS. 2019. The Interplay Between Nonalcoholic Fatty Liver Disease and Atherosclerotic Heart Disease. Hepatology. 69 (4): 1372-1374. DOI:10.1002/hep.30410.

Prineas RJ C, R. S. \& Zhang, Z.-M. (2010). ST Segment Depression (4-Codes) and Negative TWaves (5-Codes). In The Minnesota Code Manual of Electrocardiographic Findings (pp. 6097). London: Springer London.

Rautaharju PM, Surawicz B, Gettes LS, Bailey JJ, Childers R, Deal BJ, Gorgels A, Hancock EW, Josephson M, Kligfield P, Kors JA, Macfarlane P, Mason JW, Mirvis DM, Okin P, Pahlm O, 
van Herpen G, Wagner GS, \& Wellens H. 2009. AHA/ACCF/HRS recommendations for the standardization and interpretation of the electrocardiogram: part IV: the ST segment, T and U waves, and the QT interval: a scientific statement from the American Heart Association Electrocardiography and Arrhythmias Committee, Council on Clinical Cardiology; the American College of Cardiology Foundation; and the Heart Rhythm Society. Endorsed by the International Society for Computerized Electrocardiology. J Am Coll Cardiol. 53 (11): 982991. DOI:10.1016/j.jacc.2008.12.014.

Rivero D, Alhamaydeh M, Faramand Z, Alrawashdeh M, Martin-Gill C, Callaway C, Drew B, \& Al-Zaiti S. 2019. Nonspecific electrocardiographic abnormalities are associated with increased length of stay and adverse cardiac outcomes in prehospital chest pain. Heart and Lung. 48 (2): 121-125. DOI:10.1016/j.hrtlng.2018.09.001.

Setia MS. 2016. Methodology Series Module 3: Cross-sectional Studies. Indian Journal of Dermatology. 61 (3): 261-264. DOI:10.4103/0019-5154.182410.

Sigurdsson E, Sigfusson N, Sigvaldason H, \& Thorgeirsson G. 1996. Silent ST-T changes in an epidemiologic cohort study--a marker of hypertension or coronary heart disease, or both: the Reykjavik study. Journal of the American College of Cardiology. 27 (5): 1140-1147. DOI:10.1016/0735-1097(95)00614-1.

Targher G, Day CP, \& Bonora E. 2010. Risk of cardiovascular disease in patients with nonalcoholic fatty liver disease. New England Journal of Medicine. 363 (14): 1341-1350. DOI:10.1056/NEJMra0912063.

Targher G, Day CP, \& Bonora E. 2010. Risk of cardiovascular disease in patients with nonalcoholic fatty liver disease. The New England journal of medicine. 363 (14): 1341-1350. DOI:10.1056/NEJMra0912063.

Targher G, Mantovani A, Pichiri I, Rigolon R, Dauriz M, Zoppini G, Morani G, Vassanelli C, \& Bonora E. 2013. Non-alcoholic fatty liver disease is associated with an increased prevalence of atrial fibrillation in hospitalized patients with type 2 diabetes. Clinical Science (London, England: 1979). 125 (6): 301-309. DOI:10.1042/cs20130036. 
407 Targher G, Valbusa F, Bonapace S, Bertolini L, Zenari L, Pichiri I, Mantovani A, Zoppini G, 408 Bonora E, Barbieri E, \& Byrne CD. 2014. Association of nonalcoholic fatty liver disease with 409 QTc interval in patients with type 2 diabetes. Nutrition, Metabolism, and Cardiovascular 410 Diseases. 24 (6): 663-669. DOI:10.1016/j.numecd.2014.01.005.

411 Targher G, Valbusa F, Bonapace S, Bertolini L, Zenari L, Pichiri I, Mantovani A, Zoppini G, 412 Bonora E, Barbieri EJN, Metabolism, \& Diseases C. 2014. Association of nonalcoholic fatty 413 liver disease with QTc interval in patients with type 2 diabetes. 24 (6): 663-669.

414 Walsh JA, 3rd, Prineas R, Soliman EZ, Liu K, Ning H, Daviglus ML, \& Lloyd-Jones DM. 2013. 415 Association of isolated minor non-specific ST-segment and T-wave abnormalities with 416 subclinical atherosclerosis in a middle-aged, biracial population: Coronary Artery Risk 417 Development in Young Adults (CARDIA) study. Eur J Prev Cardiol. 20 (6): 1035-1041. 418 DOI:10.1177/2047487312460017.

419

420 


\section{Figure 1}

\section{Flowchart of participants selection}

ECG: Electrocardiogram; FPG: fasting plasma glucose; BMI: body mass index.

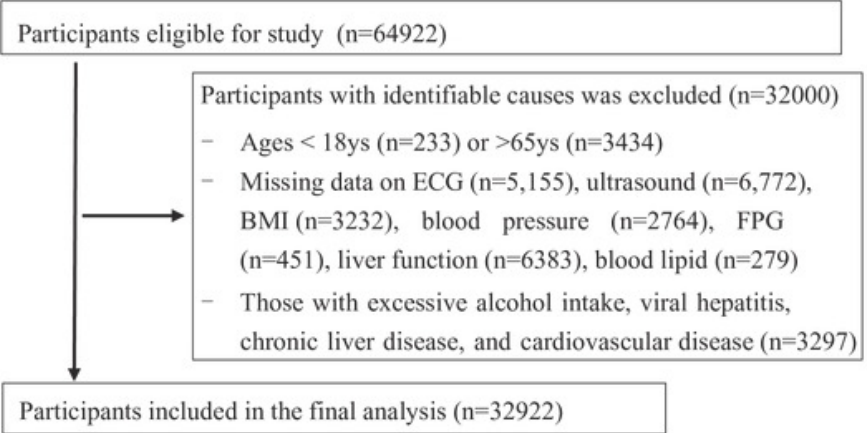


Figure 2

Proportions of non-specific ST-T segment changes in patients with mild NAFLD, moderate/severe NAFLD and without NAFLD.

$P<0.001$ for trend by the chi-squared test. 


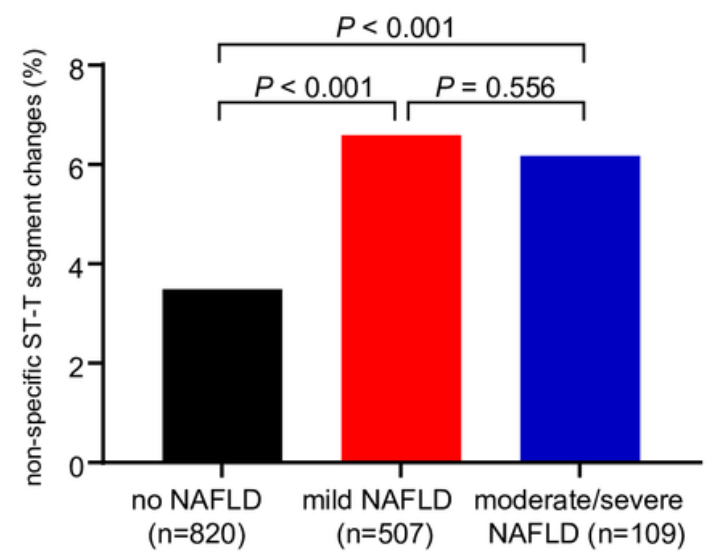


Figure 3

Logistic regression models for NAFLD as a predictor for non-specific ST-T segment changes in different subgroups. 


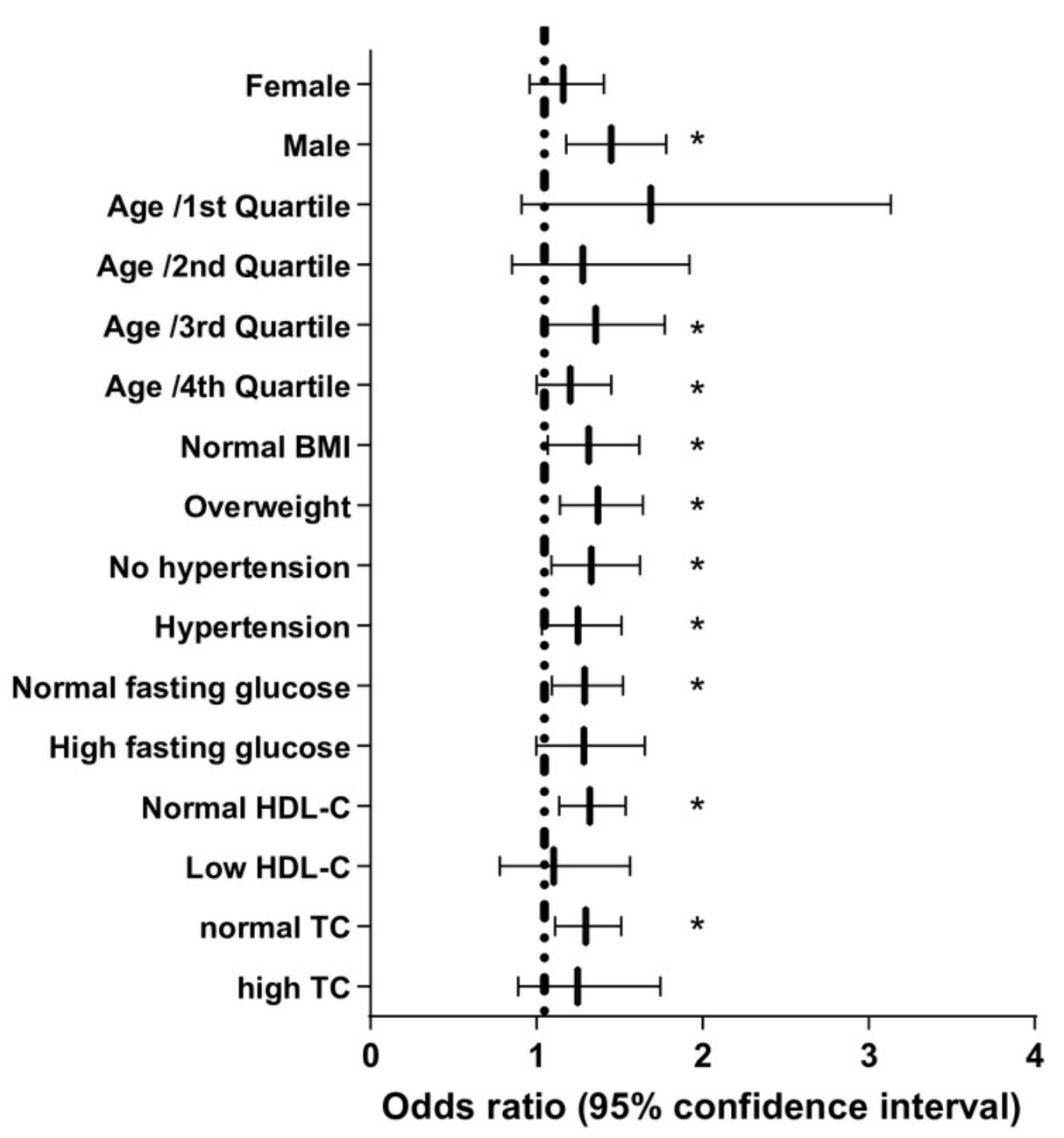

OR $\quad 95 \% \mathrm{Cl}$

$1.161(0.958 \sim 1.406)$

1.449 (1.178 1.781)

1.688 (0.909 3.134)

$1.279(0.852 \sim 1.921)$

1.356 (1.037 1.773)

$1.204(1.000 \sim 1.450)$

1.314 (1.067 1.619)

1.369 (1.142 1.641)

1.329 (1.089 1.623)

1.250 (1.033 1.512)

1.289 (1.093 1.522)

1.285 (0.999 1.653)

$1.322(1.137 \sim 1.537)$

1.103 (0.778 1.564)

1.296 (1.113 1.509)

$1.247(0.891 \sim 1.745)$

Odds ratio (95\% confidence interval) 


\section{Table $\mathbf{1}$ (on next page)}

Clinical characteristics of participants with electrocardiogram and non-specific ST-T segment changes.

Sample size, $n=32922$. Data are expressed as mean \pm SD or median (quartile). BMI: body mass index; HDL-C: high density lipoprotein cholesterol; LDL-C: low density lipoprotein cholesterol, ALT: alamine aminotransferase, AST: aspartate aminotransferase, ALP: alkaline phosphatase, GGT: gamma-glutamyl transferase. 


\begin{tabular}{|c|c|c|c|c|}
\hline & Normal & $\begin{array}{c}\text { Non-specific ST-T } \\
\text { segment changes }\end{array}$ & \multirow{2}{*}{ Chi-square } & \multirow{2}{*}{$P$-value } \\
\hline & $\begin{array}{c}\mathrm{n}(\%) \text { or } \\
\text { median(quartile) }\end{array}$ & $\begin{array}{c}\mathrm{n}(\%) \text { or } \\
\text { median(quartile) }\end{array}$ & & \\
\hline Sex & & & 108.506 & $<0.001$ \\
\hline Female(n) & $14701(46.7)$ & $872(60.7)$ & & \\
\hline Male(n) & $16785(53.3)$ & $564(39.3)$ & & \\
\hline Age (years) & $41.0(32.0-49.0)$ & $50.0(42.0-57.0)$ & -26.345 & $<0.001$ \\
\hline Height (cm) & $167.0(160.0-171.5)$ & $163.5(157.5-170.5)$ & -11.856 & $<0.001$ \\
\hline Weight (kg) & $64.5(56.0-73.8)$ & $65.4(57.2-75.5)$ & -3.862 & $<0.001$ \\
\hline $\mathrm{BMI}\left(\mathrm{kg} / \mathrm{m}^{2}\right)$ & $23.4(21.2-25.7)$ & $24.7(22.4-27.1)$ & -13.733 & $<0.001$ \\
\hline Heart rate (bpm) & $74.0(69.0-80.0)$ & $74.0(69.0-81.0)$ & -0.304 & 0.761 \\
\hline Systolic blood pressure (mmHg) & $117.0(106.0-128.0)$ & $125.0(110.0-140.0)$ & -18.430 & $<0.001$ \\
\hline Diastolic blood pressure (mmHg) & $75.0(70.0-80.0)$ & $80.0(74.0-90.0)$ & -20.054 & $<0.001$ \\
\hline Fasting plasma glucose (mmol/l) & $4.9(4.6-5.3)$ & $5.1(4.8-5.7)$ & -12.591 & $<0.001$ \\
\hline Triglyceride (mmol/l) & $1.2(0.8-1.8)$ & $1.4(1.0-2.1)$ & -10.355 & $<0.001$ \\
\hline AST (U/L) & $21.0(17.0-26.0)$ & $22.0(18.0-28.0)$ & -6.610 & $<0.001$ \\
\hline $\operatorname{ALT}(\mathrm{U} / \mathrm{L})$ & $21.0(15.0-32.0)$ & $23.0(16.0-35.0)$ & -4.651 & $<0.001$ \\
\hline $\operatorname{ALP}(\mathrm{U} / \mathrm{L})$ & $68.0(57.0-82.0)$ & $73.0(59.0-89.0)$ & -8.351 & $<0.001$ \\
\hline GGT (U/L) & $20.0(14.0-33.0)$ & $22.0(15.3-38.0)$ & -5.967 & $<0.001$ \\
\hline Total cholesterol (mmol/l) & $4.8(4.2-5.3)$ & $5.1(4.5-5.7)$ & -12.130 & $<0.001$ \\
\hline HDL-C (mmol/l) & $1.4(1.3-1.6)$ & $1.4(1.3-1.7)$ & -2.848 & 0.004 \\
\hline LDL-C (mmol/l) & $2.5(2.1-3.1)$ & $2.7(2.2-3.3)$ & -8.615 & $<0.001$ \\
\hline NAFLD severity on ultrasound (\%) & & & 148.115 & $<0.001$ \\
\hline No steatosis & $22648(71.9)$ & $820(57.1)$ & & \\
\hline Mild steatosis & $7181(22.8)$ & $507(35.3)$ & & \\
\hline Moderate/severe steatosis & $1657(5.3)$ & $109(7.6)$ & & \\
\hline
\end{tabular}




\section{Table 2 (on next page)}

Logistic regression models for NAFLD as a predictor for non-specific ST-T segment changes.

Sample size, $\mathrm{n}=32922$. Data are expressed as OR (95\% Cl). OR: odds ratio; $\mathrm{Cl}$ : confidence interval; BMI: body mass index; FPG: Fasting plasma glucose; TC: total cholesterol; HDL-C: high density lipoprotein cholesterol. Covariates included in multivariable regression models were as follows: model 1: unadjusted; model 2: age, sex; model 3: adjustment for heart rate, hypertension (blood pressure $\geq 140 / 90 \mathrm{mmHg}$ ) and fasting plasma glucose and plus the same variables included in model 2; model 4: adjustment for the same variables included in model 3 plus BMI, triglyceride, total cholesterol, HDL-C. 


\begin{tabular}{|c|c|c|c|c|}
\hline Logistic regression model & & & OR $(95 \% \mathrm{CI})$ & $P$-value \\
\hline \multicolumn{5}{|l|}{ NAFLD (yes vs no) } \\
\hline & Model 1 & & $1.925(1.729-2.143)$ & $<0.001$ \\
\hline & Model 2 & & $1.935(1.725-2.170)$ & $<0.001$ \\
\hline & Model 3 & & $1.596(1.415-1.800)$ & $<0.001$ \\
\hline & Model 4 & & $1.289(1.122-1.480)$ & $<0.001$ \\
\hline \multicolumn{5}{|c|}{ Other independent predictors of non-specific ST-T segment changes in Model 4} \\
\hline & \multicolumn{4}{|l|}{ Sex } \\
\hline & & Female & Reference & \\
\hline & & Male & $0.359(0.317 \sim 0.405)$ & $<0.001$ \\
\hline & Age & & $1.058(1.052 \sim 1.064)$ & $<0.001$ \\
\hline & BMI & & $1.072(1.050 \sim 1.093)$ & $<0.001$ \\
\hline & \multicolumn{4}{|l|}{ Hypertension } \\
\hline & & No & Reference & \\
\hline & & Yes & $1.837(1.622 \sim 2.081)$ & $<0.001$ \\
\hline & \multicolumn{4}{|l|}{ FPG } \\
\hline & & Normal & Reference & \\
\hline & & High & $1.258(1.104 \sim 1.434)$ & 0.001 \\
\hline & \multicolumn{4}{|l|}{$\mathrm{TC}$} \\
\hline & & Normal & Reference & \\
\hline & & high & $1.195(1.021-1.397)$ & 0.026 \\
\hline & \multicolumn{4}{|l|}{ HDL-C } \\
\hline & & Normal & Reference & \\
\hline & & Low & $0.839(0.711-0.989)$ & 0.037 \\
\hline
\end{tabular}

